\title{
Overexpression of CEP72 Promotes Bladder Urothelial Carcinoma Cell Aggressiveness via Epigenetic CREB-Mediated Induction of SERPINE1
}

XiangDong Li,${ }^{* \dagger}$ Pei Dong, ${ }^{* \dagger}$ WenSu Wei, ${ }^{* \dagger}$ LiJuan Jiang, ${ }^{* \dagger}$ ShengJie Guo, ${ }^{* \dagger}$ ChaoWen Huang, ${ }^{{ }^{*}}$ ZeFu Liu,${ }^{* \dagger}$ JieWei Chen, ${ }^{*{ }^{\star}}$ FangJian Zhou, ${ }^{* \dagger}$ Dan Xie, ${ }^{* \ddagger}$ and ZhuoWei Liu ${ }^{* \dagger}$

From the State Key Laboratory of Oncology in South China, ${ }^{*}$ Collaborative Innovation Center for Cancer Medicine, the Department of Urology, ${ }^{\dagger}$ and the Department of Pathology, ${ }^{\ddagger}$ Sun Yat-sen University Cancer Center, Guangzhou, Republic of China

\author{
Accepted for publication \\ February 21, 2019. \\ Address correspondence to \\ ZhuoWei Liu, M.D., Ph.D., \\ Department of Urology, Sun \\ Yat-sen University Cancer \\ Center, No. 651, Dongfeng Rd. \\ E., Guangzhou 510060, Re- \\ public of China; or Dan Xie, \\ M.D., Ph.D., State Key Labo- \\ ratory of Oncology in South \\ China, Cancer Center, Sun Yat- \\ Sen University, No. 651, \\ Dongfeng Rd. E., 510060 \\ Guangzhou, Republic of Chi- \\ na. E-mail: liuzhw@sysucc. \\ org.cn or xiedan@sysucc.org. \\ cn.
}

\begin{abstract}
A vital constituent of the centrosome involved in regulating the activity of the organelle during the cell cycle is centrosomal protein (CEP)-72, whose function in the case of human cancer yet lacks clarity. The expression dynamics of CEP72 and its clinical impact were examined in a large cohort of bladder tissues. Several experiments at both the in vitro and in vivo levels on urothelial carcinoma of the bladder (UCB) cells were conducted to understand the role of this molecule along with the mechanisms. Overexpression of CEP72 in UCB was linked with the acquisition of an aggressive phenotype, which was associated with poor prognosis. In UCB cell lines, knockdown of CEP72 using shRNA was sufficient to inhibit cell invasiveness/metastasis, whereas ectopic overexpression of CEP72 promoted cell invasiveness and/or metastasis both in vitro and in vivo. CEP72 was demonstrated to induce UCB cell aggressiveness via up-regulation of an important target downstream, the serpin family member 1 gene (SERPINE1) (alias plasminogen activator inhibitor, PAI1), ultimately leading to increased cancer cell invasiveness. Particularly, overexpression of CEP72 was associated with a sizable increase in CAMP response element-binding protein binding at the SERPINE1 promoter, leading to increased SERPINE1 transcription. Such observations are suggestive of the potential use of CEP72 as a therapeutic tool for UCB. (Am J Pathol 2019, 189: 1284-1297; https:// doi.org/10.1016/j.ajpath.2019.02.014)
\end{abstract}

Urothelial carcinoma of the bladder (UCB) is one of the most common and fatal urothelial malignancies if not treated optimally. Each year, approximately 74,000 patients in the United States and $>430,000$ patients worldwide are diagnosed with UCB. ${ }^{1,2}$ Despite advancements in surgical techniques and the design of new drugs, close to $50 \%$ patients with UCB show fatal metastases. ${ }^{3}$ The prevention of UCB metastasis is important for a favorable patient outcome. Prevention calls for an understanding of the mechanisms and molecules involved to discover new targets for therapeutic intervention that can advance the development of new treatment approaches for UCB.

The centrosome is the main microtubule-organizing center and is a crucial factor involved in cell division in mammals. ${ }^{4}$ Localized at the centrosome, centrosomal proteins (CEPs) typically regulate centrosome-associated functions. ${ }^{5}$ Centrosomal abnormalities include supernumerary centrioles, abnormal centrosome numbers and volumes, and altered phosphorylation of CEPs; the modification and accumulation of pericentriolar material are closely associated with the development of human cancers. ${ }^{6-8}$ Consequently, mutations or changes in CEP expression may likely also contribute to the development of cancer. ${ }^{4,9}$

Chromosomal aberrations of UCB have been extensively analyzed by comparative genomic hybridization, with several amplified regions, including $5 p$ or parts of $5 p$, being

Supported by Natural Science Foundation of China grants 81772716 and 81472385 and Science and Technology Planning Project of Guangdong Province grant 2017B020227007.

X.D.L., P.D., and W.S.W. contributed equally to this work.

Disclosures: None declared. 
Table 1 Association of CEP72 Expression with Clinicopathologic Characteristics of UCB Patients

\begin{tabular}{|c|c|c|c|c|}
\hline \multirow[b]{2}{*}{ Variables } & \multirow[b]{2}{*}{$\begin{array}{l}\text { Patient cases } \\
(n=221)\end{array}$} & \multicolumn{2}{|c|}{ CEP72 expression, $n(\%)$} & \multirow[b]{2}{*}{$\begin{array}{l}P \\
\text { value }\end{array}$} \\
\hline & & $\begin{array}{l}\text { Normal expression } \\
(n=140)\end{array}$ & $\begin{array}{l}\text { Overexpression } \\
(n=81)\end{array}$ & \\
\hline$\leq 60$ years & 121 & $72(59.5)$ & $49(40.5)$ & \\
\hline$>60$ years & 100 & $68(68.0)$ & $32(32.0)$ & \\
\hline Sex & & & & 0.368 \\
\hline Tumor multiplicity & & & & 0.260 \\
\hline Unifocal & 120 & $72(60.0)$ & $48(40.0)$ & \\
\hline Multifocal & 101 & $68(67.3)$ & $33(32.7)$ & \\
\hline Smoking history & & & & 0.761 \\
\hline No & 98 & $61(62.2)$ & $37(37.8)$ & \\
\hline pT status & & & & 0.014 \\
\hline pT1, Tis, Ta & 43 & $34(79.1)$ & $9(20.9)$ & \\
\hline pT2 & 74 & $51(68.9)$ & $23(31.1)$ & \\
\hline pT3 & 57 & $31(54.4)$ & $26(45.6)$ & \\
\hline pT4 & 47 & $24(51.1)$ & $23(48.9)$ & \\
\hline pN status & & & & 0.018 \\
\hline $\mathrm{pN}^{-}$ & 172 & $116(67.4)$ & $56(32.6)$ & \\
\hline $\mathrm{pN}^{+}$ & 49 & $24(49.0)$ & $25(51.0)$ & \\
\hline
\end{tabular}

Values in bold indicate significant correlation (Pearson $\chi^{2}$ test).

CEP, centrosomal protein; Ta, noninvasive papillary carcinoma; Tis, carcinoma in situ; UCB, urothelial carcinoma of bladder.

reported. ${ }^{10,11}$ This finding implies that human chromosome $5 \mathrm{p}$ contains oncogenes related to tumorigenesis and/or progression of human UCB. Several candidate oncogenes have been isolated from 5p, including CEP72, CDH6, and hTERT. ${ }^{12,13}$ CEP72 protein plays a vital role in the maintenance of the microtubule-organizing activity as well as in the strength of the centrosome structure ${ }^{14}$ CEP72 recruits key proteins to centrosomes and acts through the centrosomal microtubule-nucleation activity. ${ }^{14,15}$ Findings from a recent report suggest that the overexpression of CEP72 is linked with negative regulation of the tumor-suppressor gene $B R C A 1$ in colorectal cancer cells. ${ }^{16}$ In in a report on lung cancer, the amplification of CEP72 was frequently detected and may contribute to early stage lung tumorigenesis. ${ }^{12}$ These findings suggest that high levels of CEP72 likely drive the progression and pathogenesis of various human cancers. However, the oncogenic potential of CEP72 and its direct role in the development and pathogenesis of UCB have not been yet elucidated.

In this study, the clinicopathologic and prognostic significance of the levels of CEP72 in UCB in a large cohort of patients was examined. In addition, we evaluated the tumorigenicity of CEP72 and assessed its oncogenic potential and the associated molecular mechanisms underlying UCB.

\section{Materials and Methods}

\section{Patients and Specimen Characteristics}

The records of Department of Pathology, Sun Yat-sen University Cancer Center (SYSUCC; Guangzhou, China) were the source of formalin-fixed, paraffin-embedded tissues from 221 UCB patients subjected to radical cystectomy in the timeframe of February 2000 and March 2014. The clinical and pathologic features of the patients are listed in Table 1; the follow-up time was between 2 and 178 months, with 59 months as the median value. Before surgery, none of the patients was in receipt of systemic chemotherapy or pelvic irradiation. A review was conducted in retrospect on the patient medical records, with a focus on the survival aspect, calculated using the date of cystectomy to the date of UCB-related death or the final follow-up. Patients who died of unknown causes were excluded from this study. In addition, 10 fresh samples in pairs were acquired in 2017: UCB along with the neighboring healthy mucosa of the bladder. The standards of the eighth edition of the tumor node metastases cancer staging system from the Union Internationale Contre le Cancer (2017) was utilized to score the stage of the tumors. To ensure that protein analysis was performed on diseased and 
not healthy tissue, hematoxylin and eosin-stained sections were used to guide dissection of the UCB tissue samples, yielding samples that comprised a minimum of $70 \%$ tumor cells. The ethics committee of SYSUCC reviewed and approved the protocols of the experiments that involved these samples of human origin.

\section{UCB Cell Lines}

This work involved the use of eight cell lines of the bladder: T24, 5637, UMUC3, J82, RT4, 1, and SV-HUC-1 (normal cell line), which were sourced from ATCC (Manassas, VA) along with Biu-87 and EJ (both gifts from the First Affiliated Hospital, Sun Yat-sen University). T24, RT4, 5637, EJ, Biu-87, and SV-HUC-1 were cultured, whereas J82 and UMUC3 involved the use of RPMI 1640 and Dulbecco's Modified Eagle Medium (InvitroGen, Carlsbad, CA), both with $10 \%$ fetal bovine serum (HyClone, Logan, UT). Culture conditions were set at $37^{\circ} \mathrm{C}$, with $5 \% \mathrm{CO}_{2}$ in an atmosphere that was humidified.

\section{Western Blot Analysis}

Entire tissue and cell lysates in equal proportions were subjected to resolution on SDS-PAGE followed by electrotransfer to polyvinylidene difluoride membranes (Pall Corporation, Port Washington, NY). This method was followed by incubation with primary antibodies against CEP72, serine protease inhibitor (serpin) peptidase inhibitor, clade $\mathrm{E}$ (nexin, plasminogen activator inhibitor type 1), member 1 (SERPINE1), cAMP response element-binding protein (CREB), and mouse double minute 2 homologue (MDM2) (Sigma, St. Louis, MO); insulin-like growth factor 1, CXCL12, and melanoma cell adhesion molecule (Cell Signaling Technology, Beverly, MA); and $\beta$-catenin, E-cadherin, fibronectin, $\mathrm{N}$-cadherin, and vimentin (Becton Dickinson Transduction Laboratories, Franklin Lakes, NJ). The detection of signals involved the use of an enhanced chemiluminescence kit (Amersham Biosciences, Uppsala, Sweden). All of the experiments were performed in accordance with prescribed protocols of the manufacturer.

\section{IHC Analysis}

This study involved the use of the standard streptavidinbiotin-peroxidase complex approach. ${ }^{17}$ After preparation of $5-\mu \mathrm{m}$ tissue sections, incubation was done with 1:100 antiCEP72 or 1:200 anti-SERPINE1 or 1:100 anti-MDM2 (all from Sigma-Aldrich, St. Louis, MO) and $4^{\circ} \mathrm{C}$ storage overnight. Diaminobenzidine containing the EnVision staining system (Agilent Technologies, Santa Clara, CA) was utilized in the staining. Mouse or rabbit IgG was utilized in the negative control in place of the primary antibody.

Each protein was scored on a semiquantitative basis with a record of the positive areas and intensity of staining.
A staining index with values between 0 and 12 was calculated as follows: a score of 0 to 3 based on the intensity of staining [0 (negative); 1 (weak); and 3 (strong)] multiplied by a score of 1 to 4 based on the percentage of positive cells in the immunostaining $[1(\leq 10 \%) ; 2(>10 \%$ to $50 \%)$; 3 ( $>50 \%$ to $75 \%)$; and $4(>75 \%)$. Because the 10 normal bladder mucosal tissues showed an index of $<4$ for CEP72 expression, an index between 0 and 4 was selected as normal. An index of 6 to 12 was depicted as overexpression of CEP72. When the staining indexes were 6 for SERPINE1 and 4 for MDM2 in tumor cells, these cutoff values were considered as high levels of these proteins. Two pathologists (J.W.C. and D.X.) performed the scoring independently in a blinded approach without knowledge of the clinical data or pathology.

\section{Construction of the Recombinant Lentiviral Vector}

The $\psi$-LVRH1MP vector (GeneCopoeia, Rockville, MD) was used to construct the lentiviral vector with CEP72 shRNA in accordance with the prescribed protocol. ${ }^{18}$ The shRNA control vector (CSHCTR001-LVRU6GP; GeneCopoeia) was also included. Retroviruses carrying $\psi$-LVRU6GP-CEP72 shRNA were used to infect the T24 and EJ lines on account of high CEP72 expression. The sequences of CEP72 targeted to construct the lentiviral shRNA were: shRNA1, 5'-GCTGGATGATTTGAGACAACA-3'; and shRNA2, 5'-CCTGGTAAAGTTACATTGTCT- $3^{\prime}$. The selection of stably transfected cells was done using puromycin.

\section{Plasmid Constructs and Transfection}

Lipofectamine 2000 (InvitroGen), in accordance to prescribed protocols, was utilized to transfect $\mathrm{J} 82$ with $C E P 72$ cDNA that was subjected to PCR amplification and cloning into pcDNA3.1(+) expression vector (InvitroGen). Controls were transfected with only the vectors devoid of cDNA. Geneticin (Roche Diagnostics, Indianapolis, IN) was used for the detection of clones that expressed CEP72 stably. FuGENE HD transfection reagent (Roche Molecular Diagnostics, Pleasanton, $\mathrm{CA}$ ) was utilized to transfect UCB T24-shCEP72-1 with pcDNA-EGFP (control) or pcDNASERPINE1. Mock control was served by pcDNA-EGFP or empty vector transfections.

\section{Experimental in Vivo Metastasis Model}

After cell culture, cells in the logarithmic growth phase were trypsinized, followed by a double wash with phosphatebuffered saline:phosphate-buffered saline and centrifugation to obtain pellets. The pellet of cells was resuspended in serum-free RPMI 1640 culture medium and kept on ice until injection into mice. To evaluate metastasis in vivo, BALB/c nude mice (4 weeks of age) were obtained commercially from the Experimental Animal Center at Sun Yat-sen 
University. Each group included eight mice. In a nutshell, the tail vein of the mice was injected with $2 \times 10^{5}$ cells i.v. in a laminar flow cabinet. Six weeks after inoculation, mice were euthanized and analyzed. Mouse lung and liver paraffin sections were processed and analyzed for hematoxylin and eosin staining and immunohistochemistry analysis. These experiments were in lieu of the SYSUCC's Institutional Animal Care and Usage Committee. All animal experimental procedures were performed in accordance with the NIH's Guide for the Care and Use of Laboratory Animals. ${ }^{19}$

\section{Hematoxylin and Eosin Staining}

Tumor tissue sections were prepared as described previously. ${ }^{20}$ Nuclei were subject to staining with Mayer's hematoxylin filtered freshly for 2 minutes. After washing with water for 5 minutes, the cytoplasm, keratin, erythrocytes, and collagen were counterstained with eosin Y for 1 minute. Water was used to wash the slides, followed by dehydration through a series of graded alcohol $(60 \%, 80 \%, 99 \%$ ethanol) and cleared twice with xylol. Sections were mounted with DPX mounting medium (Sigma-Aldrich) and analyzed.

\section{Wound-Healing and Invasion Assays}

The wound-healing assay involved the use of 6-well plates to culture UCB lines until confluence, followed by scratching or wounding with the tip of a $10 \mu \mathrm{L}$ pipette. Images were recorded on an inverted light microscope (Nikon, Tokyo, Japan) at time 0 and at 24 hours after the wounding process. The assay for invasion involved the use of BD BioCoat Matrigel Invasion Chambers (Becton Dickinson) and Transwell inserts (Corning Life Sciences, Corning, New York) in accordance with the prescribed protocols of the manufacturer. Crystal violet was used to stain the Matrigel, followed by a count of the cells that invaded with microscopy. These assays were conducted in triplicate.

\section{Real-Time PCR Gene Array}

TRIzol (InvitroGen) was used to extract RNA from J82vector and J82-CEP72 cells, followed cleaning using the RNeasy MinElute Cleanup Kit (Qiagen, Valencia, CA). SuperScript III Reverse Transcriptase (InvitroGen) was utilized for reverse transcription, followed by amplification of cDNA with $2 \times$ PCR master mix (SuperArray Bioscience, Frederick, MD). Human Tumor Metastasis $\mathrm{RT}^{2}$ Profiler PCR Array (SuperArray Bioscience) with an Opticon DNA Engine ABI Prism 7900 system (Applied Biosystems, Foster City, CA) was utilized for the assay in accordance with the prescribed protocols of the manufacturer. Data were normalized to the housekeeping gene GAPDH and analyzed using the $\Delta \Delta C_{\tau}$ method.

\section{RNA Interference}

Six-well plates were used for plating UCB lines that were transfected with siRNAs against SERPINE1 and CREB designed specifically, genes with control or scrambled siRNA (Ambion, Austin, TX) using Lipofectamine 2000 Reagent (InvitroGen) in accordance to prescribed protocols. Western blot analysis was utilized to measure silencing of genes 48 hours after transfection. The sequence of SERPINE1 siRNA was 5'-AAGCAGCUAUGGGAUUCAATT- $3^{\prime}$. The sequence of $C R E B$ siRNA was $5^{\prime}$-AACTGATTCCCAAAAGCGAAG-3'.

\section{Immunofluorescence Staining}

Glass coverslips were used to reseed UCB lines until 70\% confluence. Then, cells were subjected to double-washing with phosphate-buffered saline, followed by fixation with $4 \%$ paraformaldehyde and immunofluorescence processing. The staining involved incubation with 1:100 of primary antibodies against E-cadherin and vimentin (murine) at $4^{\circ} \mathrm{C}$ overnight. After thorough washing, cells underwent 1 hour incubation with fluorescence-conjugated secondary antibody (Cy3-conjugated goat anti-mouse). This method was followed by washing and subjecting to mounting using media with DAPI (Vector Laboratories, Burlingame, CA) and observing with a fluorescence microscope (Olympus, Lake Success, NY).

\section{Chromatin Immunoprecipitation Assays}

Magna chromatin immunoprecipitation G assay kit (Millipore, Billerica, MA) was used for this assay in accordance to the prescribed protocol. TaqMan Gene Expression Master Mix (Applied Biosystems) was utilized to assess specific enrichment of the SERPINE1 promoter using the following primers: forward, 5'-GGCAGAGGGCAGAAAGGTCA-3'; and reverse, 5'-TGAACAGCCAGCGGGTCC- $3^{\prime}$ in a reaction volume of $25 \mu \mathrm{L}$. Twenty-six to 38 rounds were performed to ascertain a linear range in the reaction. Two percent agarose gel was used to resolve the products, followed by visualization using ethidium bromide. Specific antibodies against acetylation of histones 3 and 4 (AC-H3, AC-H4) were obtained from Abcam (Cambridge, MA). The assays were conducted in triplicate.

\section{Dual-Luciferase Reporter Assay}

The SERPINE1 promoter sequence was cloned to pGL3Basic plasmid. ${ }^{21}$ After lysis of J82 cells, a dual luciferase assay kit (Promega, Madison, WI) was used to analyze the activity of luciferase and Renilla adhering to the prescribed protocol. These assays were repeated two times in triplicate. Data are expressed as means \pm SD. 

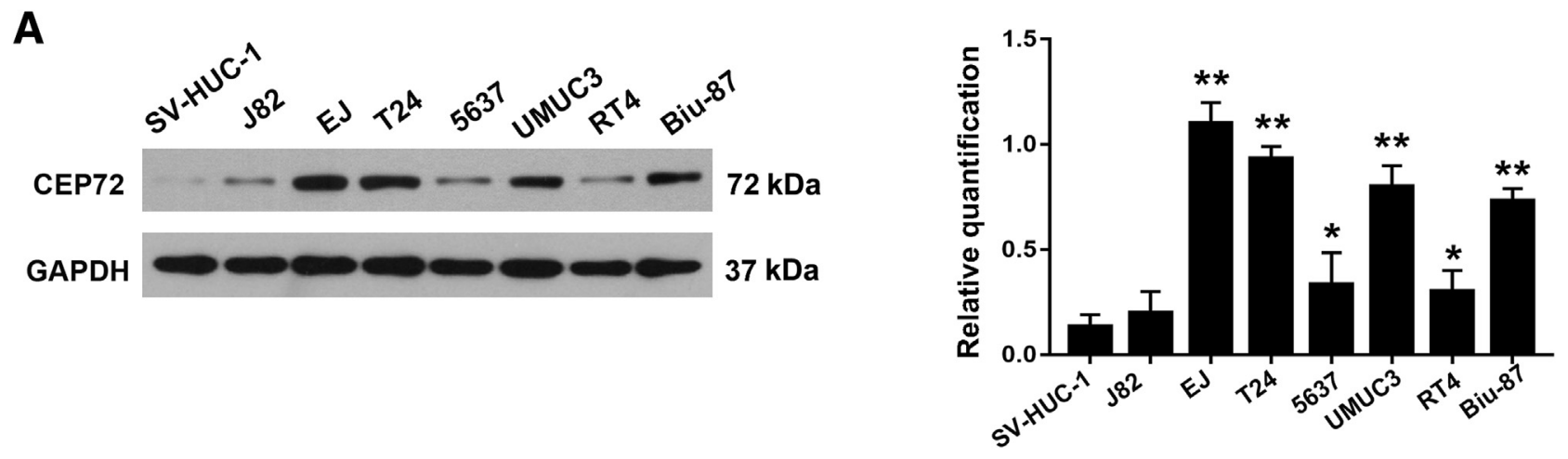

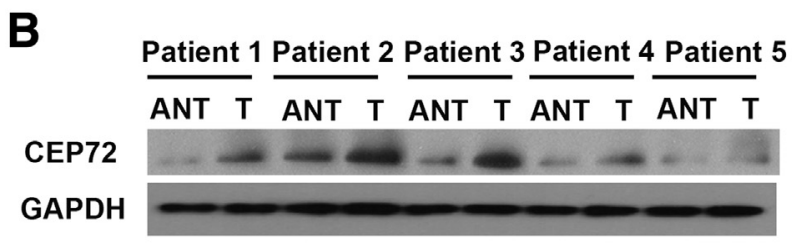

C

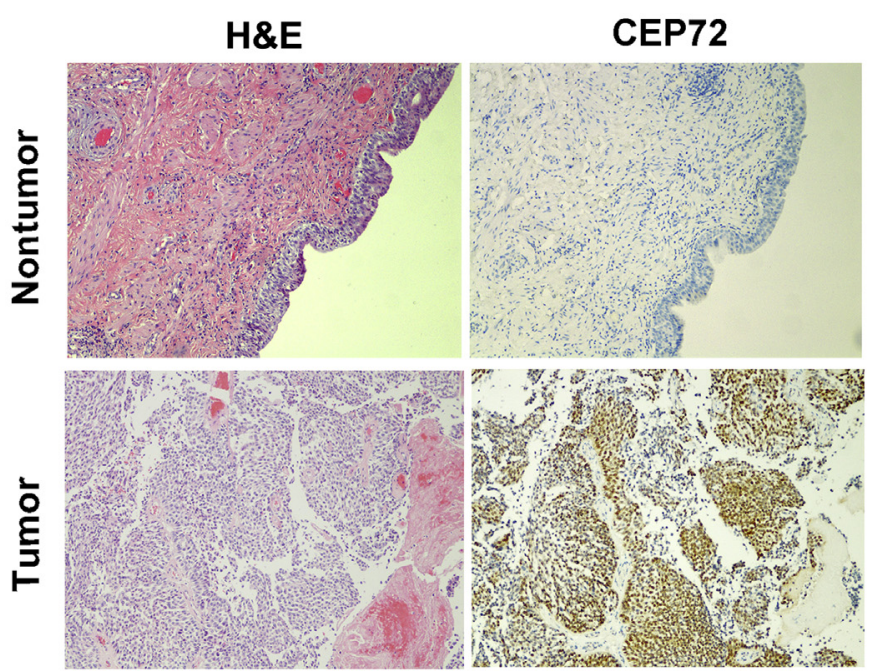

Patient 6 Patient 7 Patient 8 Patient 9 Patient 10 ANT $T$ ANT $T$ ANT $T$ ANT T ANT T

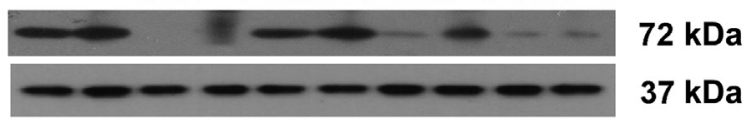

D

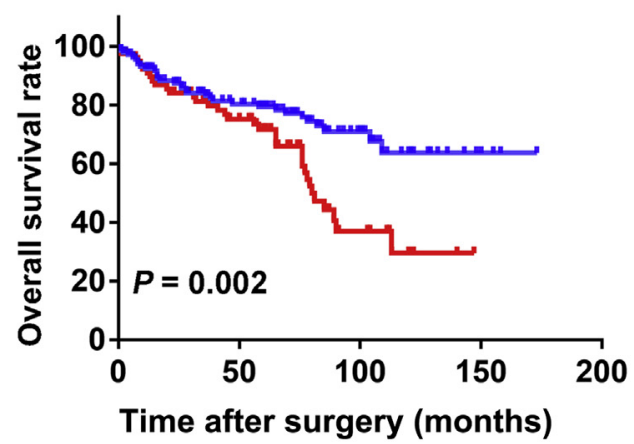

- CEP72 Normal expression
+ CEP72 Overexpression

Figure 1 Centrosomal protein (CEP)-72 expression in samples of urothelial carcinoma of the bladder (UCB). Tissues and cultured cells and significance in prognosis in patients with UCB. A: CEP72 protein is detected in seven UCB lines (Biu-87, UMUC3, EJ, 5637, T24, RT4, and J82) and one normal uroepithelial cell line (SV-HUC-1). The internal control was glyceraldehyde phosphate dehydrogenase (GAPDH). ImageJ software version 1.50i (NIH, Bethesda, MD; https://imagej. nih.gov/ij) was utilized to quantify Western blot data. B: Western blot for the amount of CEP72 in 10 paired samples of UCB and healthy bladder samples. C: Representative CEP72 immunohistochemistry staining showing normal expression in adjacent normal bladder tissue (nontumor) and overexpression in UCB (tumor) tissue (Patient 3). D: Kaplan-Meier analysis using log-rank test of the correlation between CEP72 levels and overall survival rates in 221 patients with UCB. ${ }^{*} P<0.05,{ }^{* *} P<0.01$ versus SV-HUC-1 (t-test). Original magnification, $\times 100$. ANT, adjacent non-neoplastic bladder tissue; H\&E, hematoxylin and eosin; T, carcinoma tissue.

\section{Statistical Analysis}

SPSS software version 17.0 (SPSS, Chicago, IL) was used to analyze data with the use of the Fisher exact or $\chi^{2}$ test to assess differences among variables. The Kaplan-Meier method was used for analysis of survival, and the log-rank test was used for comparison. Variable significance was assessed in the case of survival; the variables in this work were analyzed for bivariate correlations with Spearman rank correlation coefficients. All cell line data are presented as means $\pm \mathrm{SD}$. When passing the normality test, to compare two groups and multiple groups, two-tailed $t$-test and one-way analysis of variance were utilized. Statistical significance was set as $P<0.05$.

\section{Results}

CEP72 Protein Expression in Bladder Tissues and Cultured Cells

Western blot showed that among the eight bladder lines, CEP72 was expressed endogenously in the UMUC3, T24, EJ, and Biu-87 UCB cell lines, but was present only at low levels in the J82, 5637, and RT4 lines and in SVHUC-1 (the healthy line) (Figure 1A). In the bladder tissues from patients with UCB, 70\% (7/10) of the primary UCB tissues showed high levels of CEP72 (Figure 1B). 
Table 2 Univariate and Multivariate Analyses of Prognostic Parameters in 221 Patients with UCB

\begin{tabular}{|c|c|c|c|c|}
\hline Variable & \multicolumn{2}{|l|}{ Univariate analysis* } & \multicolumn{2}{|l|}{ Multivariate analysis $^{\dagger}$} \\
\hline Aged $\leq 60$ years $/>60$ years & $1.449(0.898-2.336)$ & 0.128 & $1.653(0.771-2.714)$ & 0.147 \\
\hline Smoking history & $1.410(0.864-2.303)$ & 0.170 & $1.678(0.938-3.004)$ & 0.081 \\
\hline Tumor multiplicity & $0.956(0.589-1.553)$ & 0.856 & $1.724(0.889-3.007)$ & 0.165 \\
\hline Tumor size & $1.315(0.814-2.124)$ & 0.263 & $1.075(0.654-1.766)$ & 0.776 \\
\hline pN status & $2.102(1.234-3.581)$ & 0.006 & $1.429(0.786-2.598)$ & 0.242 \\
\hline CEP72 & $1.991(1.236-3.207)$ & 0.005 & $1.710(1.050-2.783)$ & 0.031 \\
\hline
\end{tabular}

Values in bold indicate significant difference in hazard ration between the 2 groups.

${ }^{*} \chi^{2}$ test.

${ }^{\dagger}$ Cox regression model.

CEP, centrosomal protein; HR, hazard ratio; UCB, urothelial carcinoma of bladder.

CEP72 Expression in Bladder Tissues and Correlations with UCB Patient Clinicopathologic Characteristics and Survival

Immunohistochemistry analysis for CEP72 was performed on 221 formalin-fixed, paraffin-embedded UCB tissues and 10 paired non-neoplastic bladder samples for assessing whether the protein was relevant in a clinical scenario. CEP72 was virtually nil or very low in all non-neoplastic bladder tissues, whereas CEP72 was highly expressed in $36.7 \%(81 / 221)$ of the primary UCBs examined. Staining of the cell lines and normal tissues is shown in Figure $1 \mathrm{C}$. Moreover, there was a significant and positive association between CEP72 overexpression and an advanced $\mathrm{T}$ status as well as metastasis to lymph node $(P<0.05)$ (Table 1$)$. Analyses for survival showed that this overexpression served as an independent and significant factor for prognosis in the case of poor survival of patients (log-rank test, $P=0.002$, Figure 1D; multivariate Cox regression analysis, $P=0.031$, Table 2).

\section{Effects of CEP72 Expression Levels on the Aggressiveness of UCB Cultures in Vitro}

CEP72 overexpression as found on immunohistochemistry analysis showed a positive association with metastasis or advanced clinical stage of UCB. Therefore, the effect of expression of this protein on the ability of UCB lines to migrate and invade was studied. The expression of CEP72 was suppressed by two shRNAs in lines T24 and EJ, which were demonstrated to express high levels of endogenous CEP72. The efficacy of CEP72 suppression was evaluated at the level of protein with Western blot (Figure 2A). The assays for the ability of UCB lines to migrate and invade showed that both of these features were dramatically lowered in the studied cells in the case of CEP72 knockdown (Figure 2, B and C). Additionally, a J82-CEP72 cell line was designed that overexpressed CEP72 in a stable manner to check whether oncogenicity could be enhanced by this increased CEP72 ectopically (Figure 2D). Enforced CEP72 overexpression in the J82 line was associated with a considerable increase in the ability of cells to migrate and invade (Figure 2, E and F). Thus the aggressiveness of these cancers is likely linked to a vital role of increased CEP72.

\section{Effects of Overexpression of Cep72 on the Metastatic Potential of UCB Cells in Vivo}

An in vivo model was constructed to assess the role of Cep72 overexpression in an animal model. Sixteen BALB/c nude mice (eight in two groups) received J82-CEP72 or vector-only injections (as controls) in their tail veins. At 6 weeks after injection, animals were sacrificed and metastatic tumor nodule formation was examined in livers and lungs. Tumor nodule formation was not detected in any of the examined livers regardless of the experimental group, but they were found in the lungs, where overexpression of Cep72 was associated with a significantly increased frequency of J82-cell lung metastasis $(P<0.01$ ) (Figure $2 \mathrm{G}$ ). Histopathologic analysis confirmed leakage or extravasation and growth of J82-CEP72 tumors as the causes of these lesions in the lungs (Figure 2G).

\section{Effects of CEP72 on Epithelial-Mesenchymal Transition in UCB Lines}

As invasion and metastasis involve epithelial-mesenchymal transition, the potential induction of this phenomenon by CEP72 was examined. Western blot indicated an increase in the two markers in epithelium, E-cadherin and $\beta$-catenin, whereas those for mesenchymal lines - fibronectin, vimentin, and N-cadherin - were reduced after knockdown of CEP72 in the T24 and EJ lines (Figure 3A). Additionally, after overexpression of CEP72 in J82, the reverse was observed, with the amounts of the former dropping and the latter rising (Figure 3A). The reduction in E-cadherin and rise in vimentin 


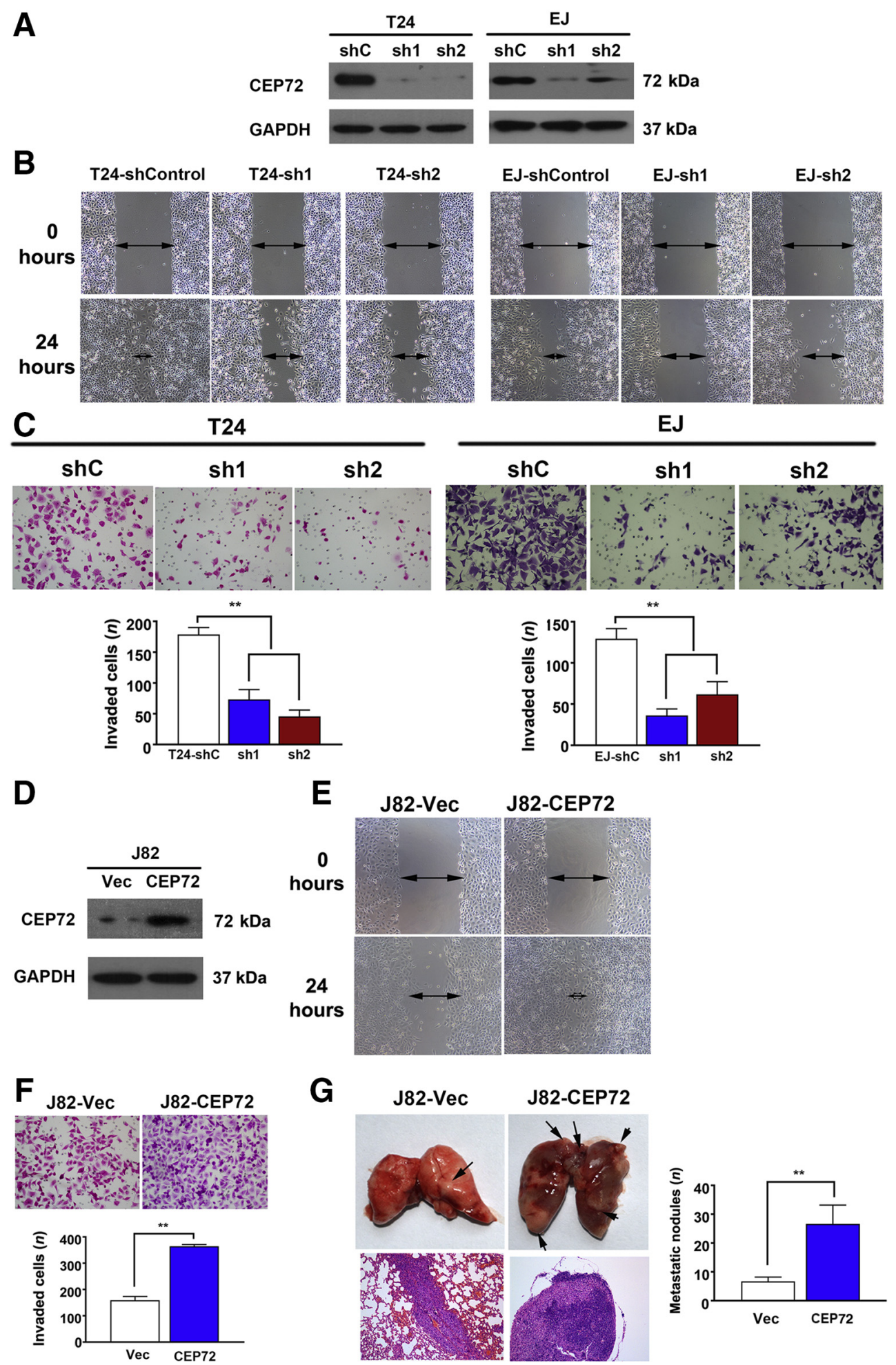

Figure 2 Centrosomal protein (CEP)-72 protein levels drive the in vitro and in vivo aggressiveness of the urothelial carcinoma of the bladder. A: Western blot confirms efficient knockdown of CEP72 protein. in T24 and EJ UCB cells using CEP72-shRNA-1 (sh1) or CEP72-shRNA-2 (sh2). The internal control was glyceraldehyde phosphate dehydrogenase (GAPDH; control). B and C: Assays for wound-healing and invasion show that CEP72-silenced T24 and EJ cells have a lowered ability to migrate and invade against respective controls (shC). The arrows show the mean relative opening distances after wound formation. D: Western blot of CEP72 protein in J82-CEP72 and control J82-vector (Vec) cells. E and F: Assays for wound-healing (E) and invasion (F) indicate increased abilities to invade and migrate as compared to controls. The arrows show the mean relative opening distances after wound formation. G: Representative images of mouse lungs with metastatic nodules (arrows) attribute to J82-Vec and J82-CEP72 cells injected into BALB/c mice (top row). Hematoxylin and eosin staining of these tumors (bottom row). The numbers of nodules at 6 weeks after the injection of J82-Vec and J82-CEP72 are shown. Data are expressed as means \pm SD from triplicate analysis. $n=8$ mice per group (G). ${ }^{* *} P<0.01$ ( $t$-test). Original magnification, $\times 100$ (B and $\mathbf{E}$, and $\mathbf{G}$, bottom row); $\times 200(\mathbf{C}$ and $\mathbf{F})$. 


$\frac{\text { T24 }}{\operatorname{shC} \operatorname{sh} 1 \text { sh2 }} \frac{\text { EJ }}{\text { shC sh1 sh2 }} \frac{\text { J82 }}{\text { Vec CEP72 }}$

\section{E-cadherin \\ $\beta$-catenin \\ Fibronectin \\ Vimentin \\ N-cadherin \\ GAPDH}
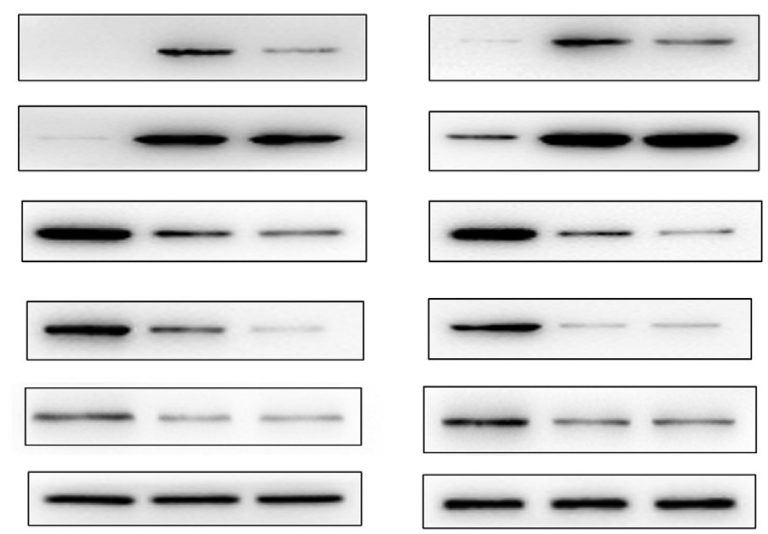

B

DAPI E-cadherin Merge
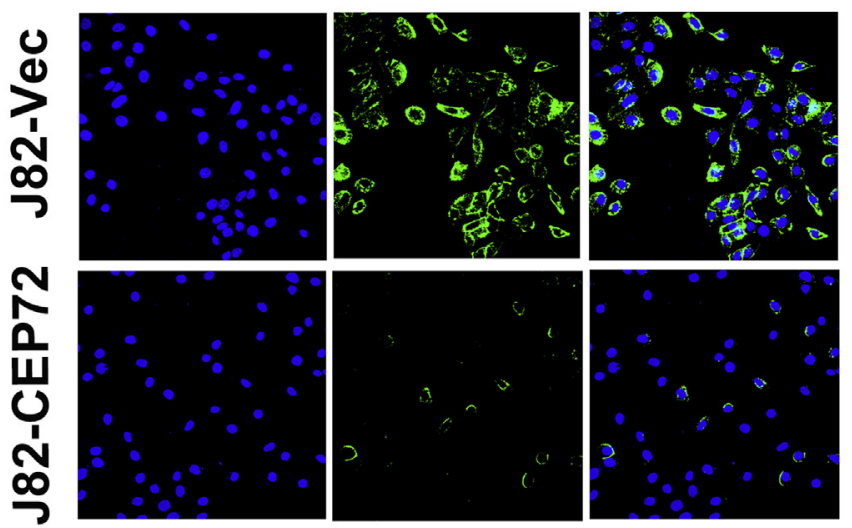

Figure 3 Centrosomal protein (CEP)-72 induces epithelial-mesenchymal transition in urothelial carcinoma of the bladder. A: Western blot analysis reveals that knock down of CEP72 by shRNAs resulted in an increased expression of E-cadherin and $\beta$-catenin and a decreased expression of fibronectin and vimentin in both T24 and EJ cells, compared with that in control shC treated cells. Decreased levels of E-cadherin and $\beta$-catenin and increased levels of fibronectin and vimentin were examined in J82-CEP72 cells compared with that in control J82-vector cells. B: Immunofluorescence for the same cells: E-cadherin (green) and vimentin (red) with nuclear DAPI stain (blue). Original magnification, $\times 600$ (B).

were established by immunofluorescence in these cells (Figure 3B). These findings are indicative of epithelial-mesenchymal transition influenced by CEP72 expression in UCB.

Effects of CEP72 Expression Levels on BRCA1 Levels in UCB Cells

A recent study identified that CEP72 overexpression was associated with inhibition of the tumor-suppressor gene BRCAl in the colorectal cancer cells. ${ }^{16}$ However, no apparent differences in the levels of breast cancer protein (BRCA)-1 between the J82-CEP72 and control J82-vector cells were observed (Supplemental Figure S1). Moreover, BRCA1 protein expression level was not impacted by the knockdown of CEP72 in T24 (Supplemental Figure S1). Such observations likely exclude the possibility that CEP72 directly regulates BRCA1 levels in UCB cells.

CEP72 Regulation of SERPINE1 Expression in UCB Cells

The expression of mRNA of J82-CEP72 against control J82-vector was subject to comparison with a PCR array of 84 genes related to metastasis to identify targets functioning in invasion/metastasis that are downstream of CEP72. SERPINE1, IGF1, and MCAM were induced, whereas CXCL12 and MDM2 were suppressed in J82-CEP72 cells by at least two-fold compared to control J82-vector cells (Supplemental Table S1 and Figure 4A). Changes in gene expression were subsequently confirmed by protein 
A

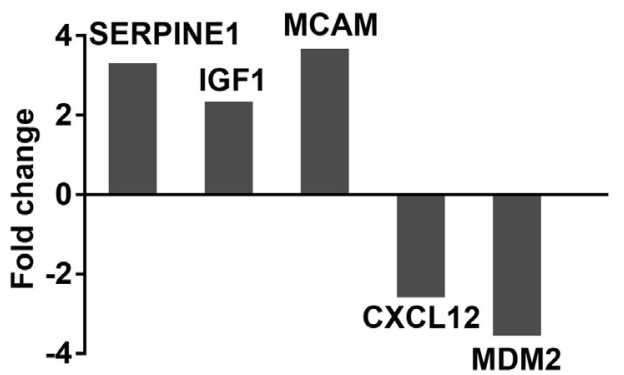

B

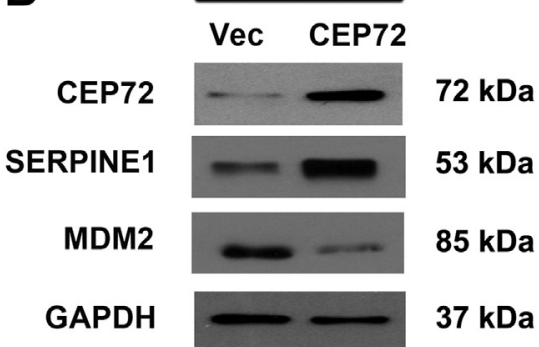

C

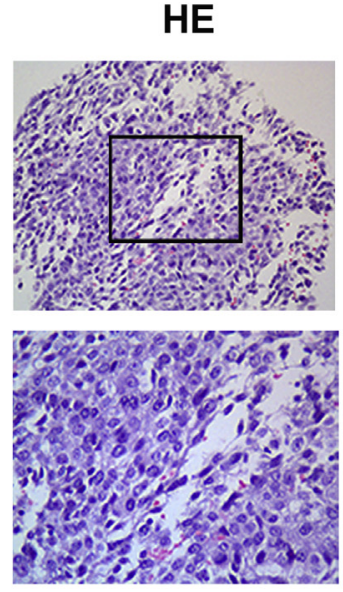

CEP72

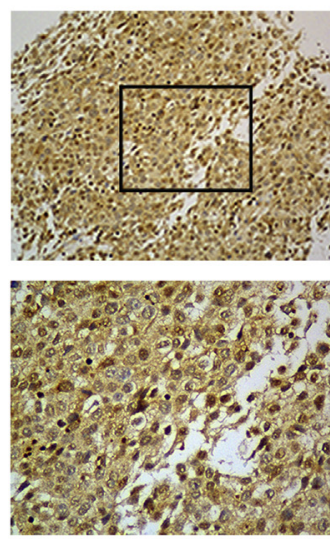

SERPINE1
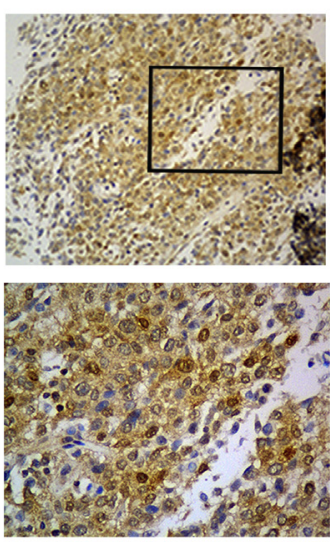

D

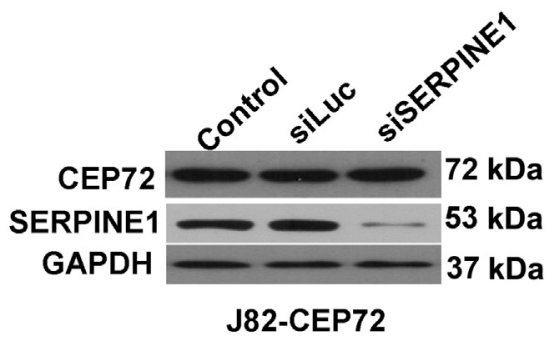

E

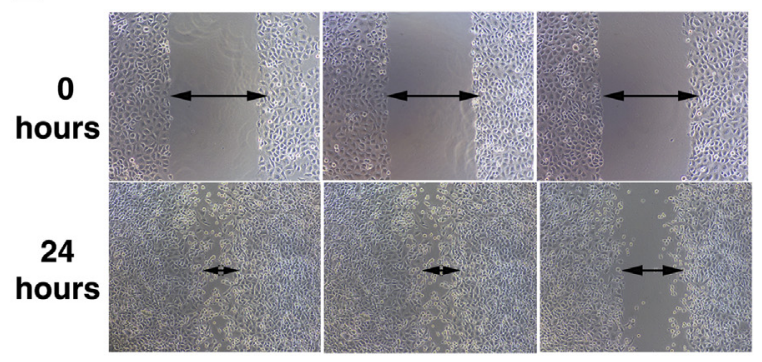

F
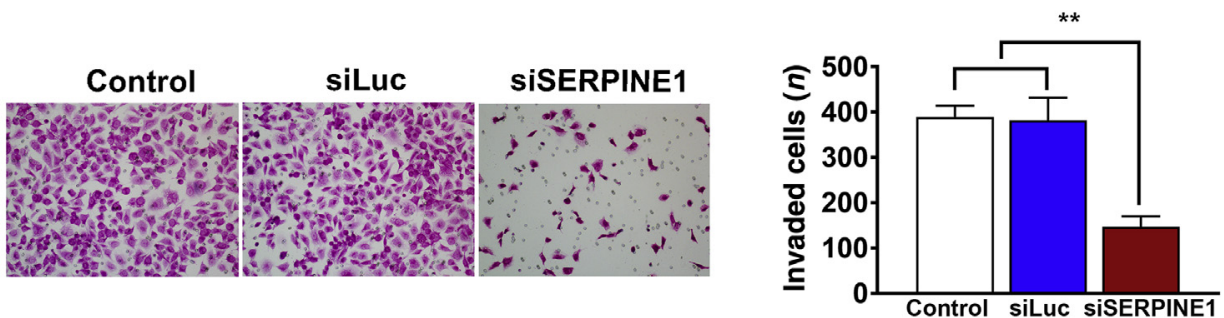

Figure 4 Centrosomal protein (CEP)-72 regulates SERPINE1 expression in urothelial carcinoma of the bladder (UCB) cells. A: Five genes, SERPINE1, IGF1, MCAM, CXCL12, and MDM2, showing at least 2-fold induction in J82-CEP72 cells compared to control J82-vector obtained from the Human Tumor Metastasis RT ${ }^{2}$ Profiler PCR Array. B: Western blot analysis of CEP72, SERPINE1, mouse double minute 2 homologue (MDM2), and glyceraldehyde phosphate dehydrogenase (GAPDH) protein levels in J82-CEP72 cells and control J82-vector (Vec) cells. C: Hematoxylin and eosin staining, CEP72 and SERPINE1 immunohistochemistry in tissue samples from a UCB patient (case 116). The boxed areas are shown at higher magnification below. D: siRNA specifically targeting SERPINE1 were transfected into J82-CEP72 UCB cells. The cells incubated with Opti-MEM (Gibco Laboratories, Gaithersburg, MD) were set as a mock control. Western blot analysis reveals efficient knockdown of SERPINE1 by J82-CEP72 cells. E and F: Assays for wound-healing (E) and invasion (F) reveal that SERPINE1 knockdown causes a sizable reduction in J82-CEP72 cells in the ability to migrate and invade. The arrows show the mean relative opening distances after wound formation. Data are expressed as means \pm SD from triplicate analysis. ${ }^{*} P<0.01$ by (t-test). Original magnification: $\times 100(\mathbf{E}) ; \times 200(\mathbf{C}$, top row, and $\mathbf{F}) ; \times 400$ (C, bottom row). Luc, luciferase; siLuc, control siRNA targeting the photinuspyralis luciferase genes. 


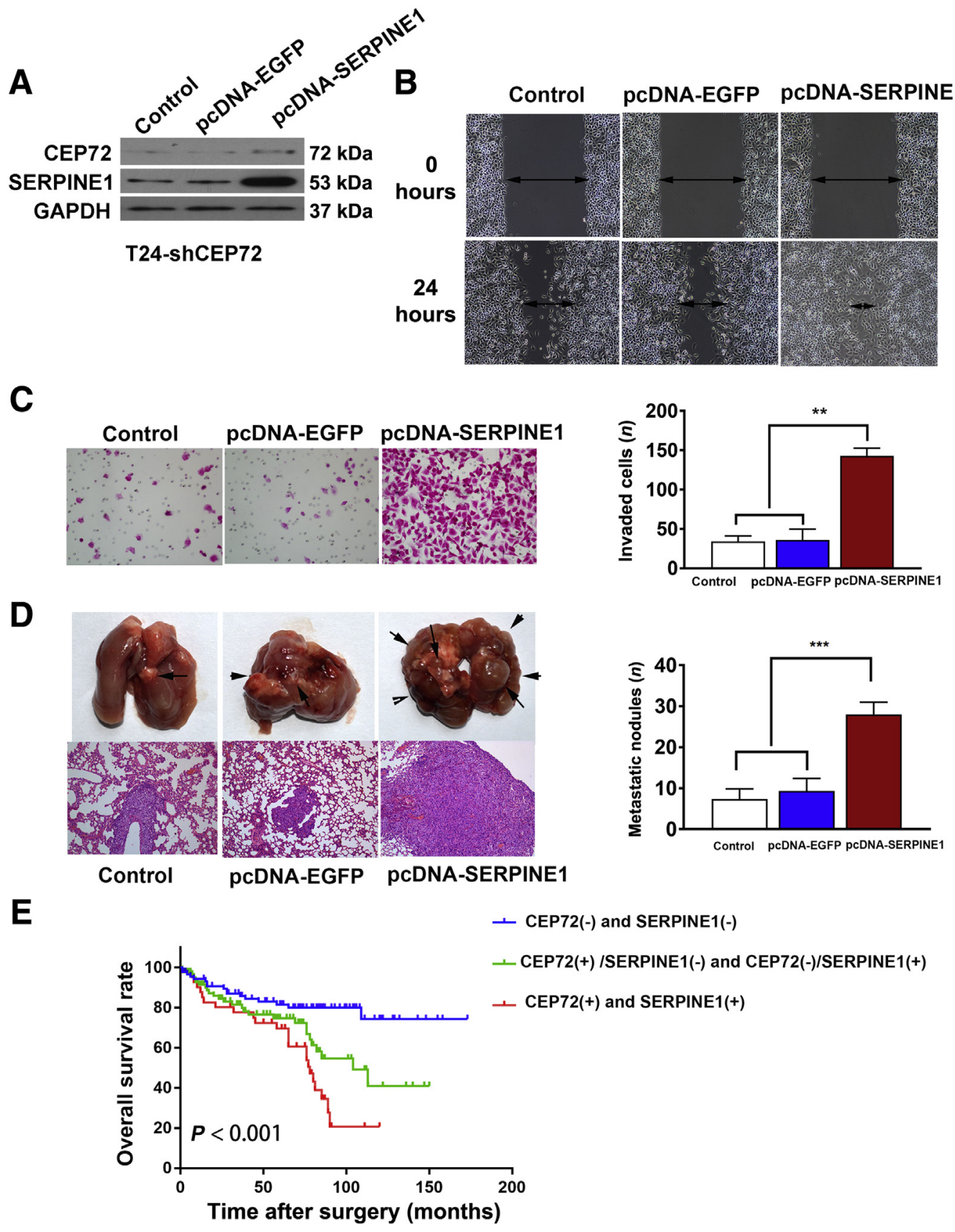

Figure 5 SERPINE1 drives centrosomal protein (CEP)-72-induced urothelial carcinoma of the bladder (UCB) cell invasiveness. A: Western blot analysis shows SERPINE1 overexpression ectopically by pcDNA-SERPINE1 transfection in T24-shCEP72 cells. B and C: Assays for wound-healing (B) and invasion (C) reveal a sizable increase in the ability of T24-shCEP72 cells to migrate and invade. The arrows show the mean relative opening distances after wound formation. D: Representative images of mouse lungs with metastatic nodules (arrows and arrowheads) originating from T24-shCEP72 (control), T24-shCEP72EGFP, and T24-shCEP72-SERPINE1 cells injected into BALB/c mice (top row). Hematoxylin and eosin staining of metastatic tumors of mouse lung (bottom row). The numbers of nodules are shown at 6 weeks after the injection. E: Kaplan-Meier analysis of data from 221 UCB cases showed that patients with low CEP72 levels in combination with low SERPINE1 levels had the best outcomes, whereas patients with high CEP72 levels in combination with high SERPINE1 levels had the worst prognosis. Data are expressed as means \pm SD from triplicate analysis. $n=8$ mice per group (D). ${ }^{* *} P<0.01,{ }^{* * *} P<0.001$ (analysis of variance). Original magnification, $\times 100($ B and D); $\times 200$ (C). GAPDH, glyceraldehyde phosphate dehydrogenase.

detection using Western blot. In agreement with the gene array, SERPINE1 was higher, whereas MDM2 was lower, in CEP72-overexpressing J82 cells (Figure 4B).

Furthermore, a significant positive correlation between CEP72 and SERPINE1 expression was identified in the cohort of UCB tissues $(P=0.012$ ) (Figure 4C and Supplemental Table $\mathrm{S} 2$ ). The difference in MDM2 expression between the tissue groups with normal expression and overexpression of CEP72 was not significant $(P=0.184)$ (Supplemental Table S2). 
A

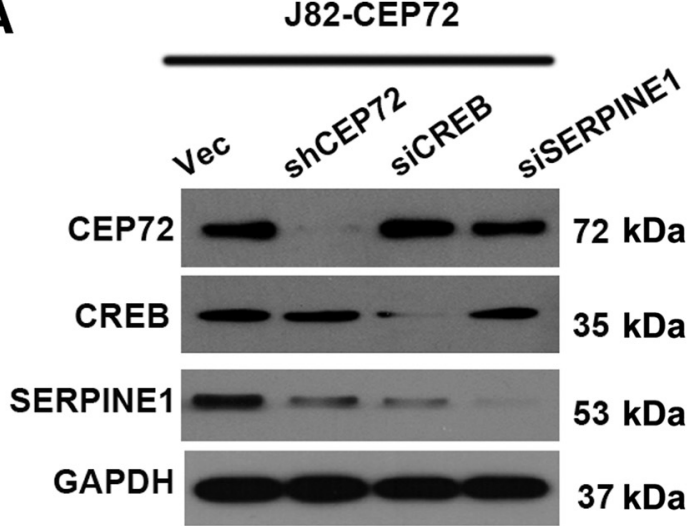

B

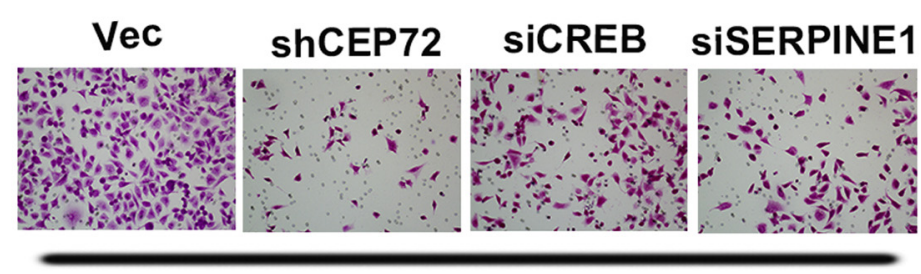

J82-CEP72
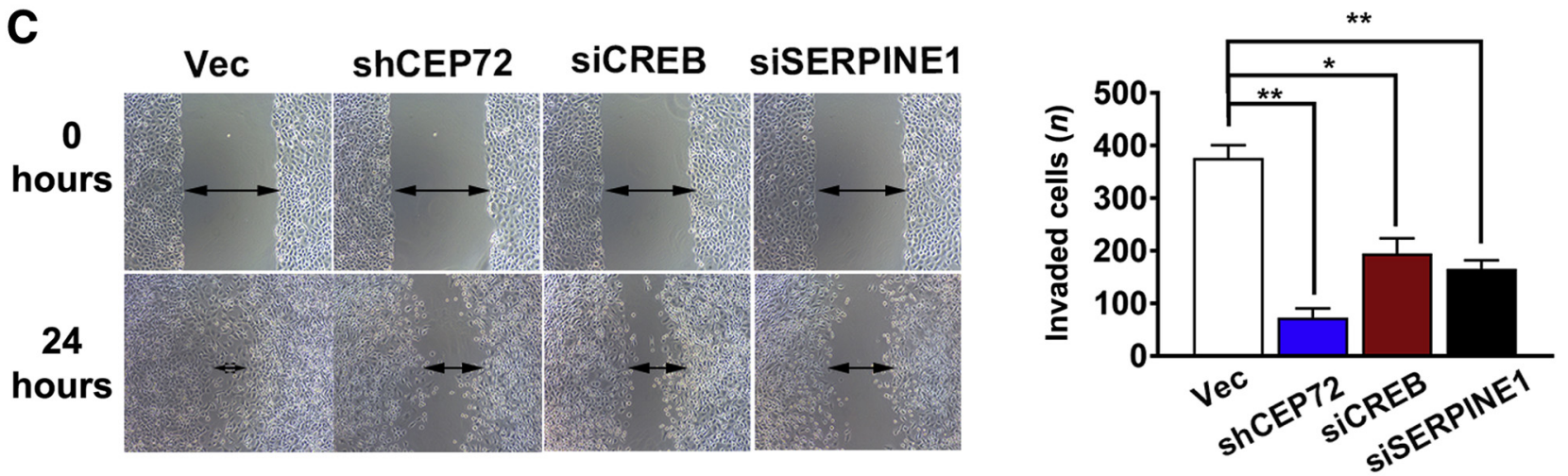

D

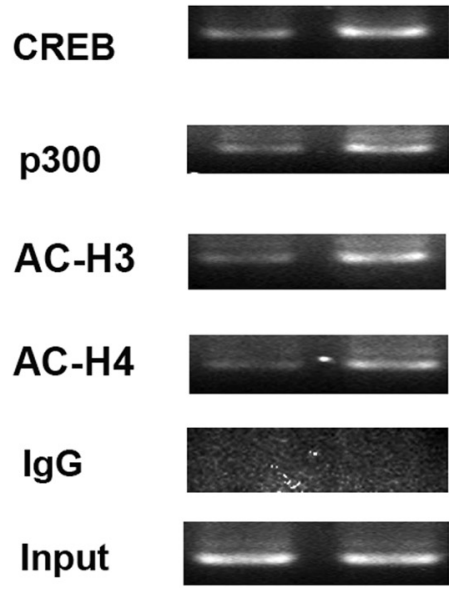

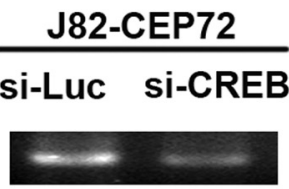
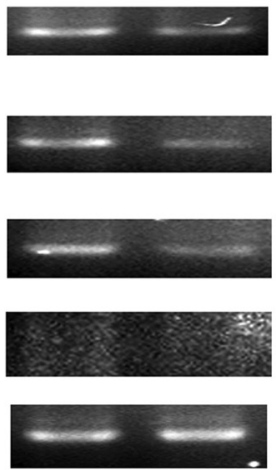

E

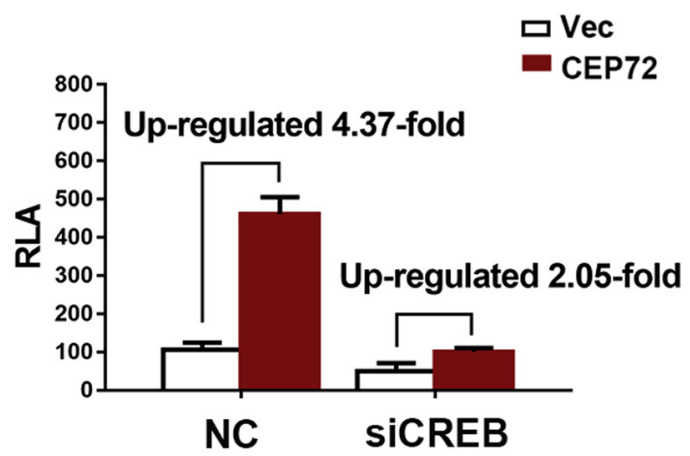

Figure 6 Induction of SERPINE1 by centrosomal protein (CEP)-72 in urothelial carcinoma of the bladder cells is partially dependent on cAMP response element-binding protein (CREB), and CEP72-mediated cell aggressiveness is recovered after CREB silencing. A: Protein expression analysis in J82-CEP72 cells transfected with ShCEP72, siCREB, or siSERPINE1 by Western blot. The decrease in expression of CEP72 leads to phenotypic changes similar to CREB knockdown. B and C: Assays for wound-healing (B) and invasion (C) in J82-CEP72 cells transfected with ShCEP72, SiCREB, or SiSERPINE1. The arrows show the mean relative opening distances after wound formation. D and E: Chromatin immunoprecipitation assays were performed to analyze enrichment of CREB and p300 on the SERPINE1 promoter after CREB silencing in J82 cells. J82 cells were first transfected with control siRNA or siCREB. After 36 hours, the cells were cotransfected with the pGL3-SERPINE1 promoter luciferase (Luc), pRL-TK Renilla luciferase construct, and control plasmid pcDNA3.1(+) or pcDNA-CEP72 plasmid. Luciferase activity at the SERPINE1 promoter was measured and normalized relative to luciferase activity (RLA). Data are expressed as means \pm SD from three independent experiments. ${ }^{*} P<0.05,{ }^{*} P<0.01$ (analysis of variance). Original magnification, $\times 200(\mathbf{B}) ; \times 100($ C). AC-H, acetylation of histones; $\mathrm{GAPDH}$, glyceraldehyde phosphate dehydrogenase; NC, negative control; Vec, vector. 
Effects of SERPINE1 on CEP72-Induced UCB Cell Aggressiveness

To assess the involvement of SERPINE1 in the CEP72induced aggressiveness of UCB, a rescue experiment was performed. SERPINE1 was subjected to knockdown using RNAi expression in J82-CEP72 (Figure 4D). The ability to migrate (in terms of speed) and invade, as shown by healing and invasion assays, was reduced after siSERPINE1 treatment (Figure 4, E and F). Furthermore, when SERPINE1 was introduced into CEP72-silenced T24 cells (Figure 5A), inhibition of UCB cell aggressiveness in vitro and metastatic ability in vivo were substantially ameliorated (Figure 5, B-D). Next, it was assessed in patient samples whether high expression of both the CEP72 and SERPINE1 proteins directly influenced overall UCB patient survival rate. As expected, overall survival in patients positive for CEP72/SERPINE1 was significantly lower than that in those who were negative $(P<0.001, \log$-rank test $)$ (Figure 5E). Overall, aggressiveness in the case of UCB driven by SERPINE1 is evidenced by these observations.

\section{Up-Regulation of SERPINE1 by CEP72 in UCB Cells and Partial Dependence on CREB}

The transcription factor CREB has been shown to directly regulate SERPINE1 (ie, PAI1) levels by binding to the SERPINE1 promoter. ${ }^{21}$ We therefore investigated whether CEP72-mediated induction of SERPINE1 expression is mediated by CREB in UCB cells. In agreement with expectations, functional studies showed that CEP72-mediated induction of SERPINE1, which typically enhances migration/ invasive capacity in J82-CEP72 cells, was decreased when CREB levels were diminished (Figure 6, A-C). However, CREB protein levels were not affected after $C E P 72$ silencing (Figure 6A), suggesting that CEP72 may regulate SERPINE1 expression at the transcriptional level in UCB cells. A previous study revealed that $\mathrm{CREB}$ can interact with the protein acetyltransferase p300 to enhance transcriptional activity by increasing the amount of acetylation of histones 3 and 4 in cells of advanced prostate cancer. ${ }^{22}$ CREB along with $\mathrm{p} 300$ was enriched in J82-CEP72 of SERPINE1 promoter as shown by chromatin immunoprecipitation assay, concurrent with higher acetylation of the histones (AC-H3 and AC-H4), in comparison to controls (Figure 6D). In addition, when endogenous $C R E B$ was silenced by siRNA in the J82-CEP72 cells, the enrichment of CREB and p300 on the SERPINE1 promoter, as well as the levels of AC-H3 and AC-H4, were markedly decreased (Figure 6D). The assay for dual luciferase reporter indicated that silencing of CREB partially blocked the enhancement of CEP72 on SERPINE1 transcription (induced 4.4-fold and 2.1-fold in control and siCREB cells, respectively) (Figure 6E). Overall, these results suggest that CEP72 transcriptionally induces SERPINE1 expression via CREB in UCB cells.

\section{Discussion}

In the present study, CEP72 expression was initially examined by Western blot in a series of carcinoma and nonneoplastic bladder cells and tissues. A major amount of these samples showed endogenous CEP72 in high amounts. Immunohistochemistry staining suggested that CEP72 was frequently overexpressed in patients with UCB. Further use of statistics showed a positive association between CEP72 in these samples and the advances in the clinical stage as well as the status of the lymph node, which could serve as an independent prognostic factor of poor survival in patients. Therefore, immunohistochemistry microscopic evaluation of CEP72 expression can serve as a supplemental check to examine those patients at higher risk for either metastasis and/or recurrence. This approach may favorably predict the outcome at a clinical level in UCB patients. These findings also highlight the potentially important role of CEP72 in the development and/or progression of UCB.

A sequence of in vitro and in vivo experiments was used to evaluate the role played by CEP72 in regulating the malignancy of UCB. Enforced knockdown or ectopic overexpression of CEP72 in UCB cells substantially suppressed or promoted cell migration and invasion, respectively. An elevation at significant levels of lesions that metastasized to the lungs was seen in the mouse model when Cep72 was overexpressed. Because epithelial-mesenchymal transition plays a key function in the ability of UCB tumors to invade and metastasize, ${ }^{18,23}$ the association of this phenomenon with the aspect of CEP72 controlling movement was studied. As expected, CEP72 expression led to suppression of protein levels of many epithelial markers and increased expression of mesenchymal markers. After the discovery that CEP72 functions as a vital oncogene in UCB pathogenesis, the biology of the mechanisms of CEP72 augmenting an aggressive behavior of these cells was characterized. CEP72 is frequently induced in colorectal cancer tissues, and overexpression of CEP72 mirrors the phenotype of the tumor-suppressor gene BRCAl deletion. ${ }^{16}$ However, in the current study, no differences were observed in BRCA1 levels before or after CEP72 overexpression or knockdown. Therefore, it appears that CEP72 promotes aggressiveness in UCB cells by regulating targets and/or pathways other than those involving BRCA1, suggestive of a tumor-specific mechanism in cancer advancement by CEP72.

A significant positive correlation was identified between CEP72 and SERPINE1 expression in our cohort of UCB tissues. These results suggest that CEP72 regulates cell aggressiveness via regulation of SERPINE1 in UCB cells. SERPINE1 (or PAI1) is a serine protease inhibitor and the main regulator of the plasminogen activation system, where it has complex functions. ${ }^{24}$ Its mode of action involves inhibition of tissue- and urokinase-type plasminogen activators. The urokinase-type plasminogen activator and 
SERPINE1 coregulate each other's expression and are involved in the process of cancer metastasis, which requires proteolytic cleavage of the extracellular matrix and basement membrane. ${ }^{25}$ SERPINE1 was previously characterized as an oncogene in human cancers, including bladder, ${ }^{26,27}$ breast, ${ }^{28}$ and gastric cancers. ${ }^{29}$ SERPINE1 is predominantly involved in modulating cell migration and invasion. ${ }^{30}$ Previous studies suggest that low SERPINE1 levels are significantly correlated with reduced tumor progression. ${ }^{25,31}$ In the present study, high levels of SERPINE1 protein not only were closely associated with UCB aggressiveness and/or poor patient survival, but also were positively correlated with CEP72 protein expression. To determine whether SERPINE1 is a downstream target involved in CEP72-induced UCB cell aggressiveness, a rescue experiment was performed with J82-CEP72 cells that express high levels of CEP72 and SERPINE1. When J82-CEP72 cells were transfected with siSERPINE1, their invasive ability was significantly decreased. Furthermore, inhibition of UCB cell invasive and metastatic abilities was restored when SERPINE1 was introduced into CEP72-silenced T24 cells. These data suggest that SERPINE1 is a crucial downstream target of CEP72 and that increased SERPINE1 levels are a major driver of CEP72-induced invasiveness in UCB cells.

The molecular mechanisms by which CEP72 regulates SERPINE1 expression have not yet been elucidated. A recent publication from Dimova et $\mathrm{al}^{21}$ suggested direct binding of CREB to the SERPINE1 gene promoter to enhance transcription in human hepatoma cells. Other groups have reported that CREB may recruit the histone acetyltransferase p300 to acetylate histones ( $\mathrm{H} 3$ and $\mathrm{H} 4)$ and lead to transcriptional activation of certain target genes. $^{22}$ In the current study, enrichment of CREB on the SERPINE1 promoter was substantially enhanced by ectopic overexpression of CEP72 in J82 UCB cells, concurrent with higher amounts of acetylated $\mathrm{H} 3$ and $\mathrm{H} 4$. Although altered levels of CREB were not observed after CEP72 knockdown in J82-CEP72 cells, a reduction in CREB and p300 recruitment to the SERPINE1 promoter was observed. It is important to highlight that the luciferase reporter assays showed that silencing of CREB by siRNA in J82-CEP72 cells only partly inhibited the transcriptional activity of CEP72 on the SERPINE1 promoter, and that CEP72mediated SERPINE1 induction was partially inhibited by CREB knockdown. These data suggest that in addition to CREB, other unknown mechanisms may be involved in the CEP72-mediated regulation of SERPINE1 in UCB cells. Importantly, further work is needed to clarify the mechanisms of CEP72-mediated regulation of SERPINE1 in detail.

Our findings indicate that overexpression of $C E P 72$ as an oncogene is vital in the pathogenesis of UCB. CEP72 enhances binding of CREB to the SERPINE1 promoter, resulting in the induction of SERPINE1 and increased UCB cell aggressiveness. CEP72 has potential as a target of therapeutic intervention in human UCB.

\section{Acknowledgment}

The Biu-87 and EJ cell lines were gifted by First Affiliated Hospital, Sun Yat-Sen University.

\section{Supplemental Data}

Supplemental material for this article can be found at https://doi.org/10.1016/j.ajpath.2019.02.014.

\section{References}

1. Jacobs BL, Lee CT, Montie JE: Bladder cancer in 2010: how far have we come? CA Cancer J Clin 2010, 60:244-272

2. Kamat AM, Hahn NM, Efstathiou JA, Lerner SP, Malmstrom PU, Choi W, Guo CC, Lotan Y, Kassouf W: Bladder cancer. Lancet 2016, 388:2796-2810

3. Sternberg CN, Bellmunt J, Sonpavde G, Siefker-Radtke AO, Stadler WM, Bajorin DF, Dreicer R, George DJ, Milowsky MI, Theodorescu D, Vaughn DJ, Galsky MD, Soloway MS, Quinn DI: ICUD-EAU International Consultation on Bladder Cancer 2012: chemotherapy for urothelial carcinoma-neoadjuvant and adjuvant settings. Eur Urol 2013, 63:58-66

4. Rivera-Rivera Y, Saavedra HI: Centrosome-a promising anti-cancer target. Biologics 2016, 10:167-176

5. Zyss D, Gergely F: Centrosome function in cancer: guilty or innocent? Trends Cell Biol 2009, 19:334-346

6. Godinho SA, Pellman D: Causes and consequences of centrosome abnormalities in cancer. Philos Trans R Soc Lond B Biol Sci 2014, 369:20130467

7. Niu Y, Liu T, Tse GM, Sun B, Niu R, Li HM, Wang H, Yang Y, Ye X, Wang Y, Yu Q, Zhang F: Increased expression of centrosomal alpha, gamma-tubulin in atypical ductal hyperplasia and carcinoma of the breast. Cancer Sci 2009, 100:580-587

8. Rida PC, Cantuaria G, Reid MD, Kucuk O, Aneja R: How to be good at being bad: centrosome amplification and mitotic propensity drive intratumoral heterogeneity. Cancer Metastasis Rev 2015, 34: 703-713

9. Shi X, Li D, Wang Y, Liu S, Qin J, Wang J, Ran J, Zhang Y, Huang Q, Liu X, Zhou J, Liu M: Discovery of centrosomal protein 70 as an important player in the development and progression of breast cancer. Am J Pathol 2017, 187:679-688

10. Lopez V, Gonzalez-Peramato P, Suela J, Serrano A, Algaba F, Cigudosa JC, Vidal A, Bellmunt J, Heredero O, Sanchez-Carbayo M: Identification of prefoldin amplification (1q23.3-q24.1) in bladder cancer using comparative genomic hybridization (CGH) arrays of urinary DNA. J Transl Med 2013, 11:182

11. Lindgren D, Sjodahl G, Lauss M, Staaf J, Chebil G, Lovgren K, Gudjonsson S, Liedberg F, Patschan O, Mansson W, Ferno M, Hoglund M: Integrated genomic and gene expression profiling identifies two major genomic circuits in urothelial carcinoma. PLoS One 2012, 7:e38863

12. Kang JU, Koo SH, Kwon KC, Park JW, Kim JM: Gain at chromosomal region $5 \mathrm{p} 15.33$, containing TERT, is the most frequent genetic event in early stages of non-small cell lung cancer. Cancer Genet Cytogenet 2008, 182:1-11

13. Gugnoni M, Sancisi V, Gandolfi G, Manzotti G, Ragazzi M, Giordano D, Tamagnini I, Tigano M, Frasoldati A, Piana S, Ciarrocchi A: Cadherin-6 promotes EMT and cancer metastasis by restraining autophagy. Oncogene 2017, 36:667-677

14. Oshimori N, Li X, Ohsugi M, Yamamoto T: Cep72 regulates the localization of key centrosomal proteins and proper bipolar spindle formation. EMBO J 2009, 28:2066-2076 
15. Kodani A, Yu TW, Johnson JR, Jayaraman D, Johnson TL, AlGazali L, Sztriha L, Partlow JN, Kim H, Krup AL, Dammermann A, Krogan NJ, Walsh CA, Reiter JF: Centriolar satellites assemble centrosomal microcephaly proteins to recruit $\mathrm{CDK} 2$ and promote centriole duplication. Elife 2015, 4. 07519

16. Luddecke S, Ertych N, Stenzinger A, Weichert W, Beissbarth T, Dyczkowski J, Gaedcke J, Valerius O, Braus GH, Kschischo M, Bastians $\mathrm{H}$ : The putative oncogene CEP72 inhibits the mitotic function of BRCA1 and induces chromosomal instability. Oncogene 2016, 35: 2398-2406

17. Xie D, Lau SH, Sham JS, Wu QL, Fang Y, Liang LZ, Che LH, Zeng YX, Guan XY: Up-regulated expression of cytoplasmic clustering in human ovarian carcinoma. Cancer 2005, 103:277-283

18. Li XD, Zhang JX, Jiang LJ, Wang FW, Liu LL, Liao YJ, Jin XH, Chen WH, Chen X, Guo SJ, Zhou FJ, Zeng YX, Guan XY, Liu ZW, Xie D: Overexpression of maelstrom promotes bladder urothelial carcinoma cell aggressiveness by epigenetically downregulating MTSS1 through DNMT3B. Oncogene 2016, 35:6281-6292

19. Committee for the Update of the Guide for the Care and Use of Laboratory Animals: National Research Council: Guide for the Care and Use of Laboratory Animals: Eighth Edition. Washington, DC, National Academies Press, 2011

20. Cai MY, Tong ZT, Zheng F, Liao YJ, Wang Y, Rao HL, Chen YC, Wu QL, Liu YH, Guan XY, Lin MC, Zeng YX, Kung HF, Xie D: EZH2 protein: a promising immunomarker for the detection of hepatocellular carcinomas in liver needle biopsies. Gut 2011, 60:967-976

21. Dimova EY, Samoylenko A, Kietzmann T: FOXO4 induces human plasminogen activator inhibitor-1 gene expression via an indirect mechanism by modulating HIF-1alpha and CREB levels. Antioxid Redox Signal 2010, 13:413-424

22. Ianculescu I, Wu DY, Siegmund KD, Stallcup MR: Selective roles for cAMP response element-binding protein binding protein and p300 protein as coregulators for androgen-regulated gene expression in advanced prostate cancer cells. J Biol Chem 2012, 287 : 4000-4013

23. McConkey DJ, Choi W, Marquis L, Martin F, Williams MB, Shah J, Svatek R, Das A, Adam L, Kamat A, Siefker-Radtke A, Dinney C:
Role of epithelial-to-mesenchymal transition (EMT) in drug sensitivity and metastasis in bladder cancer. Cancer Metastasis Rev 2009, 28: 335-344

24. Placencio VR, DeClerck YA: Plasminogen activator inhibitor-1 in cancer: rationale and insight for future therapeutic testing. Cancer Res 2015, 75:2969-2974

25. Botla SK, Savant S, Jandaghi P, Bauer AS, Mucke O, Moskalev EA, Neoptolemos JP, Costello E, Greenhalf W, Scarpa A, Gaida MM, Buchler MW, Strobel O, Hackert T, Giese NA, Augustin HG, Hoheisel JD: Early epigenetic downregulation of microRNA-192 expression promotes pancreatic cancer progression. Cancer Res 2016, 76:4149-4159

26. Liu M, Li M, Liu J, Wang H, Zhong D, Zhou H, Yang B: Elevated urinary urea by high-protein diet could be one of the inducements of bladder disorders. J Transl Med 2016, 14:53

27. Chen LM, Chang M, Dai Y, Chai KX, Dyrskjot L, SanchezCarbayo M, Szarvas T, Zwarthoff EC, Lokeshwar V, Jeronimo C, Parker AS, Ross S, Borre M, Orntoft TF, Jaeger T, Beukers W, Lopez LE, Henrique R, Young PR, Urquidi V, Goodison S, Rosser CJ: External validation of a multiplex urinary protein panel for the detection of bladder cancer in a multicenter cohort. Cancer Epidemiol Biomarkers Prev 2014, 23:1804-1812

28. Foekens JA, Peters HA, Look MP, Portengen H, Schmitt M, Kramer MD, Brunner N, Janicke F, Meijer-van Gelder ME, HenzenLogmans SC, van Putten WL, Klijn JG: The urokinase system of plasminogen activation and prognosis in 2780 breast cancer patients. Cancer Res 2000, 60:636-643

29. Nishioka N, Matsuoka T, Yashiro M, Hirakawa K, Olden K, Roberts JD: Plasminogen activator inhibitor 1 RNAi suppresses gastric cancer metastasis in vivo. Cancer Sci 2012, 103:228-232

30. Kwaan HC, McMahon B: The role of plasminogen-plasmin system in cancer. Cancer Treatment Res 2009, 148:43-66

31. Gutierrez LS, Schulman A, Brito-Robinson T, Noria F, Ploplis VA, Castellino FJ: Tumor development is retarded in mice lacking the gene for urokinase-type plasminogen activator or its inhibitor, plasminogen activator inhibitor-1. Cancer Res 2000, 60: $5839-5847$ 